\title{
Acute Strength Exercise Decreases Satiety by Modifying Blood Cytokines Levels in Physically Active Men
}

\author{
Thamires Mendonça de Carvalho ${ }^{1}$ (D) , Juliana Soares Severo ${ }^{1}$ (D) , Felipe Giovanni de Sousa e Silva Santos ${ }^{1}$ (D) , \\ Ana Karolina Martins Cavalcante ${ }^{3}$ (D) , Alexandre Havt ${ }^{3}$ (i) , Armênio Aguiar dos $\operatorname{Santos}^{3}$ (D) , \\ Francisco Leonardo Torres-Leal ${ }^{1,2,4}$ (1) , Moisés Tolentino ${ }^{1,2,5}$
}

\begin{abstract}
${ }^{1}$ Universidade Federal do Piauí, Programa de Pós-Graduação em Alimentos e Nutrição, Teresina, PI, Brasil. ${ }^{2}$ Universidade Federal do Piauí, Programa de Pós-Graduação em Farmacologia, Teresina, PI, Brasil. ${ }^{3}$ Universidade Federal do Ceará, Faculdade de Medicina, Departamento de Fisiologia e Farmacologia, Fortaleza, CE, Brasil. ${ }^{4}$ Universidade Federal do Piaui, Departamento de Biofisica e Fisiologia, Teresina, PI, Brasil. ${ }^{5}$ Universidade Federal do Piaui, Departamento de Educação Física, Laboratório de Exercício e Trato Gastrintestinal, Teresina, PI, Brasil.
\end{abstract}

Editor: Angelina Zanesco. UNESP/Rio Claro, SP, Brazil

\begin{abstract}
Aim: Exercise elicits adaptations in several physiological systems, such as the gastrointestinal tract. We evaluated the effects of an acute strength exercise (acute-SE) on gastric satiety and its correlation with creatinine kinase (CK), lactate, and plasma cytokine levels in humans. Methods: Anthropometric parameters, body composition, muscular strength, and satiety (drink test protocol) at rest and exercise were assessed. Results: In the squat, bench press, and T-bar row exercises, we observed a significant decrease $(p<0.05)$ in muscular strength in the second, third, and fourth sets compared with that in the first set. Compared with rest, we observed a significant increase $(p<0.05)$ in CK and lactate levels after acute-SE. In the drink test, acute-SE significantly increased $(p<0.05)$ the total intake, calories ingested, and a total time of ingestion. Concerning cytokines, there was a significant increase $(p<0.05)$ after acute-SE of IL-1 $\beta$ and IL-6 levels at the beginning of the test and a decrease in IL- $6,-10,-13$, and TNF- $\alpha$ levels after acute-SE at the end of the test $(p<0.05)$. There was a correlation between CK, lactate, and total intake after acute-SE $(p<0.05)$ as well as between IL-6, 13, TNF- $\alpha$, and volume ingested in the last score of the drink test after acute-SE $(p<0.05)$. Conclusion: Acute-SE decreases satiety associated with changes in lactate, CK, and plasma cytokine levels in healthy humans.
\end{abstract}

Keywords: biochemical parameters; cytokines; exercise; satiety; strength training.

\section{Introduction}

Nowadays, the benefits of regular physical exercise are well known ${ }^{1}$. According to the American College of Sports Medicine, exercise is equated with medicine, since it helps prevent or ameliorate common diseases such as diabetes and arterial hypertension, besides promoting well-being and quality of life ${ }^{2}$.

Physical exercise is often prescribed for people with gastrointestinal motility problems for the amelioration of symptoms and complaints such as bloating and constipation ${ }^{3}$. However, prolonged strenuous exercise, possibly involving thermal stress, can provoke symptoms such as diarrhea, vomiting, intestinal inflammation, and gastroesophageal reflux ${ }^{4}$.

The gastrointestinal tract also plays a key role in controlling hunger and satiety. Gastric and intestinal tissues release orexigenic and anorexigenic hormones, which interact with neurotransmitters via the central nervous system (CNS), modulating satiety, and food consumption. These hormones regulate the appetite and energy intake that occur with changes in hunger perception and satiety 5 .

Acute physical exercise has an important association with fasting and eating patterns. Recently, we demonstrated in experimental models that acute exercise decreases gastric emptying and increases gastric compliance, depending on the exercise intensity ${ }^{4}$. In humans, recent evidence suggests that exercises above $75 \%$ of maximum oxygen consumption $\left(\mathrm{VO}_{2}\right.$ max) can suppress hunger by regulating appetite hormones, a phenomenon known as "exercise-induced anorexia," which may restrict energy intake ${ }^{6}$. Thus, exercise intensity appears to be an important determinant of appetite regulation in humans? In a meta-analysis, Horner et al. ${ }^{8}$ investigated the effect of acute exercise on gastric emptying and reported controversial results, because the training protocols analyzed were mostly aerobic. However, few investigations have evaluated the impact of resistance training on gastrointestinal parameters.

Strength exercise (SE) is a form of anaerobic exercise that includes voluntary contractions of the skeletal muscles of a specific body part against some external resistance, a force that opposes movement ${ }^{9}$. SE with load increments increases the strength and cross-sectional area of muscles. This process, called muscle hypertrophy, increases the size and possibly the number of myofibrils in the muscle fibers ${ }^{10}$.

Strength exercise can result in localized damage to muscle tissue. This damage can be observed in the sarcolemma, basal 
lamina as well as in the contractile elements and the cytoskeleton ${ }^{11}$. Usually, this damage is accompanied by the release of enzymes such as creatine kinase (CK), lactate dehydrogenase, and myoglobin, as well as other proteins into the blood. Serum $\mathrm{CK}$ has been proposed as one of the best indirect indicators of muscle damage, due to its ease of identification and the relatively low cost of assays to quantify it ${ }^{12}$.

Another important biochemical marker is lactate. It increases in skeletal muscle exponentially with exercise intensity, accumulating in the plasma when the rate of production exceeds the rate of removal ${ }^{13}$. This accumulation may be an important mediator of the effects of high-intensity exercise on appetite-regulating hormones. In gastric mucosal cell cultures, ghrelin-producing cells were found to contain abundant G-protein coupled receptor 81 (GPR81), which binds with lactate to inhibit ghrelin release ${ }^{14}$, suggesting lactate's role in appetite suppression. This association between lactate and GPR81 can suppress ghrelin despite the increased activity of the sympathetic nervous system. The anorexigenic effect of lactate is observed in both rats and humans, where peripheral lactate administration suppresses energy intake ${ }^{15}$. Whether exercise-induced alterations in circulating lactate directly stimulate the release of anorexigenic hormones (PYY, GLP-1, PP) is yet to be determined ${ }^{6}$.

In addition, neuromuscular and metabolic changes stimulated by SE are associated with an inflammatory response. After performing SE, pro-inflammatory cytokines are primarily released, particularly IL-6, whose levels increase fivefold after $30 \mathrm{~min}$. After this period, IL-6 stimulates the secretion of anti-inflammatory cytokines, IL-1 receptor antagonist (IL-1ra), and IL-10 by blood mononuclear cells ${ }^{16}$. These inflammatory responses are efficiently coordinated and stimulate tissue repair in skeletal muscles. Cytokines such as interleukins (IL-6, IL-10, and IL-1 $\beta$ ) and TNF- $\alpha$ are secreted by macrophages in skeletal muscles. These cytokines can influence appetite and satiety by regulating hormone secretion ${ }^{17}$.

In relation to the gastrointestinal tract, recent reports have shown that increasing interleukin IL-6 concentrations delays gastric emptying, leading to reduced postprandial glycemia ${ }^{18}$. In addition, cytokine enhancers such as IL- $1 \beta$, TNF- $\alpha$, and IL- 6 are reportedly associated with decreased gastric emptying and altered gastrointestinal functions ${ }^{19}$. Thus, we hypothesized that acute SE (acute-SE) alters satiety and food ingestion, and this phenomenon may be related to plasma cytokine levels.

\section{Methods}

\section{Sample}

This experimental study included 15 healthy males, aged 18-30 years, who practiced strength exercise for at least 3 months ( 1 hour a day and 3 days a week). Participants diagnosed with gastrointestinal tract or metabolic diseases, such as diabetes, as well as cardiovascular or musculoskeletal disorders, were excluded.

Supplementary materials S1. summarizes the experimental protocol, which consisted of three visits over 14 days. On day 0 , basal anthropometric and body composition measurements were performed, along with the first evaluation of satiety (drink test rest) and blood collection. On day 7, the 1-RM was conducted. On day 14, the acute-SE protocol, second evaluation of satiety (drink test - exercise), and blood collection were conducted. This study was approved by the Research Ethics Committee of the Federal University of Piauí (UFPI) under protocol number 1.698.573).

\section{Anthropometric Evaluation}

Two days before the evaluation, all participants received the recommendations to follow for body composition assessments. Initially, we determined body mass $(\mathrm{Kg})$ and height $(\mathrm{m})$. The body mass index (BMI) was calculated as the mass (Kg) divided by the square of the height $\left(\mathrm{m}^{2}\right)$ of the individual. Body composition evaluation was performed using the electrical bioimpedance method with an InBody S10 ${ }^{\circledR}$ tetrapolar system (DSM-BIA).

\section{One-Maximum Repetition Test (1RM)}

The muscular strength of the participants was evaluated by the 1-RM test. One repetition maximum testing in the order of bench press, squat, and T-bar row occurred one week before the experimental protocols. All participants performed a warm-up (jogging) for $5 \mathrm{~min}$ and then one set of 15 repetitions of the respective exercise at approximately $50 \%$ of the $1 \mathrm{RM}$ prior to 1 RM testing. The load was increased gradually (15\%) during the test until the participants were no longer able to complete the movement, allowing 3-5. A rest interval of 5 min was allowed between attempts ${ }^{20}$. No rest was allowed between the concentric and eccentric phases of the movement, and the participants were encouraged verbally to exert maximum effort. Two fitness professionals oversaw all testing sessions.

\section{Exercise Protocol}

After one week of the 1RM test, the participants returned to the laboratory to execute the exercise protocol. Each participant performed four sets of bench press, squat, and T-bar row. The recovery between sets was 30 seconds and the interval between exercises was 2 minutes. Each set was performed with overload equivalent to $70 \%$ of $1 \mathrm{RM}$ and the participants were encouraged to execute the maximum repetitions in each series until muscle exhaustion.

\section{Assessment of Satiety by Drinking Test}

To asse15ss satiety, we used a drinking test according to the previous study ${ }^{5}$. All fifteen participants were studied in two situations: (1) at rest (control) on day 0; and (2) just after acute-SE on day 14. Briefly, all participants were instructed to eat their last meal at 10:00 PM the previous day. At 6:00 
AM, the participants were instructed to ingest a standardized breakfast consisting of $200 \mathrm{~mL}$ of orange-flavored juice (108 Kcal, $26 \mathrm{~g}$ carbohydrates, $0.90 \mathrm{~g}$ proteins, and 0 total fats) and 1 packet of $26 \mathrm{~g}$ of salted crackers (121 Kcal, $2.2 \mathrm{~g}$ protein, 17 $\mathrm{g}$ carbohydrates, $4.8 \mathrm{~g}$ total fats). This meal was standard for all participants, considering that everyone underwent an equal period of fasting and that differences in the amounts of nutrients could lead to a misinterpretation of satiety. Two hours after eating, the participants remained at rest for another 40 minutes, corresponding to the control situation (day 0), or performed the acute strength exercise session (day 14). After both periods, the participants were requested to drink the standardized liquid meal, chocolate milk (Nescau ${ }^{\circledR}$, Nestlé, Vevey, Switzerland), containing $9.77 \mathrm{Kcal}, 1.5 \mathrm{~g}$ carbohydrates, $0.375 \mathrm{~g}$ protein, and $0.2625 \mathrm{~g}$ fat. The meal was ingested at a constant rate of 15 $\mathrm{mL} / \mathrm{min}$ as measured by a peristaltic pump. At 5-min intervals, the participants scored their satiation level by indicating verbal descriptors on a $0-5$ scale $(0=\mathrm{I}$ do not feel anything, $1=\mathrm{I}$ 'm starting to feel full, $2=$ I'm a little bit full, $3=$ I'm full, $4=$ I'm very full, 5 = I can't take it anymore). The test was completed when the participant reported a score of 5 .

\section{Blood Biochemical Analysis}

During the drink test, blood samples were collected from the antecubital vein of each participant at two different points (in first and last scoring of drink test), in both phases (at baseline and on the day of exercise). The blood samples were centrifuged at 3,500 rpm for 12 minutes at $4{ }^{\circ} \mathrm{C}$. The serum and plasma were separated into microtubes $(1.5 \mathrm{~mL})$ and were stored at $-80^{\circ} \mathrm{C}$. The blood lactate levels (mg/dL) were measured using a biotechnology kit. Creatinine kinase (CK) levels were assessed with Labteste kits using a Labmax Plenno automatic analyzer. The plasma cytokine level was assessed using the Bio-Plex Pro Human Cytokine kit and processed using the Bio-Plex 200 System $^{\circledR}$, with one analyzer per suspension assay.

\section{Statistical Analysis}

Initially, all data were analyzed for normality using the Shapiro-Wilk test with the GraphPad Prism software, version 6 (GraphPad Software, San Diego, CA). For the statistical comparison of the data obtained during repetitions of the series of acute-SE, one-way analysis of variance (ANOVA) was used for repeated measures followed by the Tukey test. The levels of cytokines at baseline and after the exercise were analyzed by two-way ANOVA, followed by the Tukey test. Linear regression analysis was conducted, and curves were plotted, starting from the single values of each group to comparison and interaction between satiety and blood cytokine levels. The results are presented in Tables 3. The Pearson linear correlation test was applied after confirming the normal distribution of the data. We used correlation to study the inter-relation between the IL-6 pro-inflammatory cytokine and anti-inflammatory cytokine IL-10 in the balance of the inflammatory process. ${ }^{*} \mathrm{p}<0.05$ was considered statistically significant.

\section{Results}

\section{Anthropometrics Parameters}

In this study the participants presentation mean values of weight $(\mathrm{kg}) 78.9 \pm 10.2$, age (years) $22.8 \pm 2.8$, estature (m) $1.7 \pm 0.07, \mathrm{BMI}(\mathrm{Kg} / \mathrm{m} 2) 25.8 \pm 2.3$, body fat $(\%) 16.15$ \pm 6.7 , fat mass $(\mathrm{kg}) 13.4 \pm 5.9$, lean mass $(\mathrm{kg}) 61.8 \pm 7.5$ and basal metabolic rate (Kcal) $1785 \pm 173.4$. In relation of the squat, bench press, T-bar row in the 1RM test, presented the mean values were $(104.0 \pm 15.7,81.3 \pm 19.71$ and $90.6 \pm 16.29 \mathrm{Kg}$ ).

Figure 1 shows the mean values of repetitions performed in the acute-SE in a squat (box A), bench press (box B), and T-bar row (box $C$ ) exercises. We observed significant decreases $(p<0.05)$ in the repetition numbers after muscle fatigue in series 2, 3, and 4 compared to the first set in all three exercises (squat, bench press, and T-bar row). In squat exercise, the mean values of the repetitions in the $1^{\text {st }}, 2^{\text {nd }}, 3^{\text {rd, }}$ and $4^{\text {th }}$ sets were $12.2 \pm 3.5 v s .8 .2 \pm 2.4,6.8 \pm 2.8$, and 5.6 \pm 2.2 , respectively. In bench press exercise, the mean values of the repetitions in the $1^{\text {st }}, 2^{\text {nd }}, 3^{\text {rd, }}$ and $4^{\text {th }}$ sets were $10.4 \pm 2.1$ vs. $7.4 \pm 1.4,5.1 \pm 1.5$, and $4.0 \pm 1.2$, respectively. In the T-bar row exercise, the mean values of the repetitions in the $1^{\text {st }}$, $2^{\text {nd }}, 3^{\text {rd, }}$ and $4^{\text {th }}$ sets were $10.7 \pm 3.7 v s .7 .8 \pm 1.9,6.8 \pm 1.5$, and $5.9 \pm 1.2$, respectively.

\section{Response of the Drink Test}

Table 1 shows the results of the drink test at two different moments: at rest and after exercise. A significant increase $(\mathrm{p}<0.05)$ in the total volume was observed after acute-SE $(1866 \pm 531.5 \mathrm{~mL})$ in comparison with the baseline value $(1410 \pm 424.0 \mathrm{~mL}$, box A). In relation to total time (box B), a significant increase $(\mathrm{p}<0.05)$ was observed after acute-SE (137.6 \pm 53.9 minutes) in comparison with baseline (104.4 \pm 34.6 minutes $)$. In box $C$, a significant increase $(\mathrm{p}<0.05)$ of ingested calories was observed after acute-SE (1193 \pm $384.0 \mathrm{kcal}$ ) in comparison with baseline values (vs. 985.9 $\pm 345.4 \mathrm{kcal})$.

\section{Plasma Cytokine Levels}

Table 2 reports the plasma levels of all pro-inflammatory, and anti-inflammatory cytokines assessed in the scores 0 and 5 ("I don't feel anything" and "I can't take it anymore"). In relation to IL-1 $\beta$, a significant increase $(p<0.05)$ was obtained after acute-SE compared with the basal state at score 0 . In the case of IL- 6 , a significant increase was found at score 0 $(p<0.05)$ and a significant decrease at score $5(p<0.05)$, both after exercise training. The values of TNF- $\alpha$ significantly decreased after acute acute-SE at score $5(\mathrm{p}<0.05)$. Considering anti-inflammatory cytokines, a significant decrease was found for plasma IL-10 and -13 after acute-SE at score $5(\mathrm{p}<0.05)$. 


\section{Linear Regression Analysis}

Table 3 shows the results of the linear regression of blood CK and lactate, inflammatory cytokines, and total baseline intake and after acute-SE from the beginning to the end of the drink test. We found a positive relationship between CK and total intake after acute-ST at score $5(\mathrm{r}=0.519, \mathrm{p}<0.05)$ and for blood lactate and total intake after acute-SE at score $0(\mathrm{r}=0.758, \mathrm{p}<0.01)$. No correlation was found between plasma cytokine levels and total intake $(\mathrm{p}>0.05)$.

In relation to the biochemical parameters. we evaluated two different satiety test scores ("I don't feel anything" and "I can't take it anymore") at rest and after the acute-SE session until muscle exhaustion. A significant increase $(p<0.05)$ was observed in the values of CK and blood lactate after acute-SE at the score "I don't feel anything" and for glucose at the score "I can't take it anymore" after the exercise session $(p<0.05)$. However, we observed a possible relationship between IL-6 and IL-10 values at rest and after exercise at two points of the drink test. At both points (scores 0 and 5 ) there was a positive correlation between the two cytokines $(\mathrm{p}<0.05)$.

\section{A)}

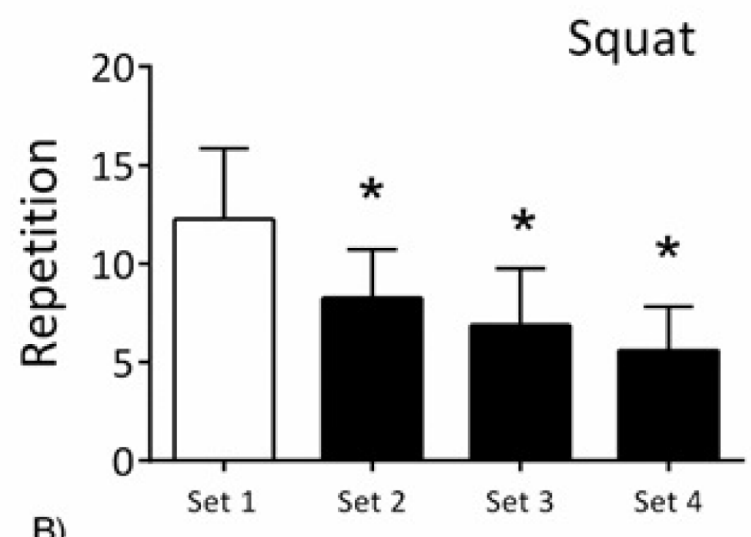

B)

\section{Bench Press}
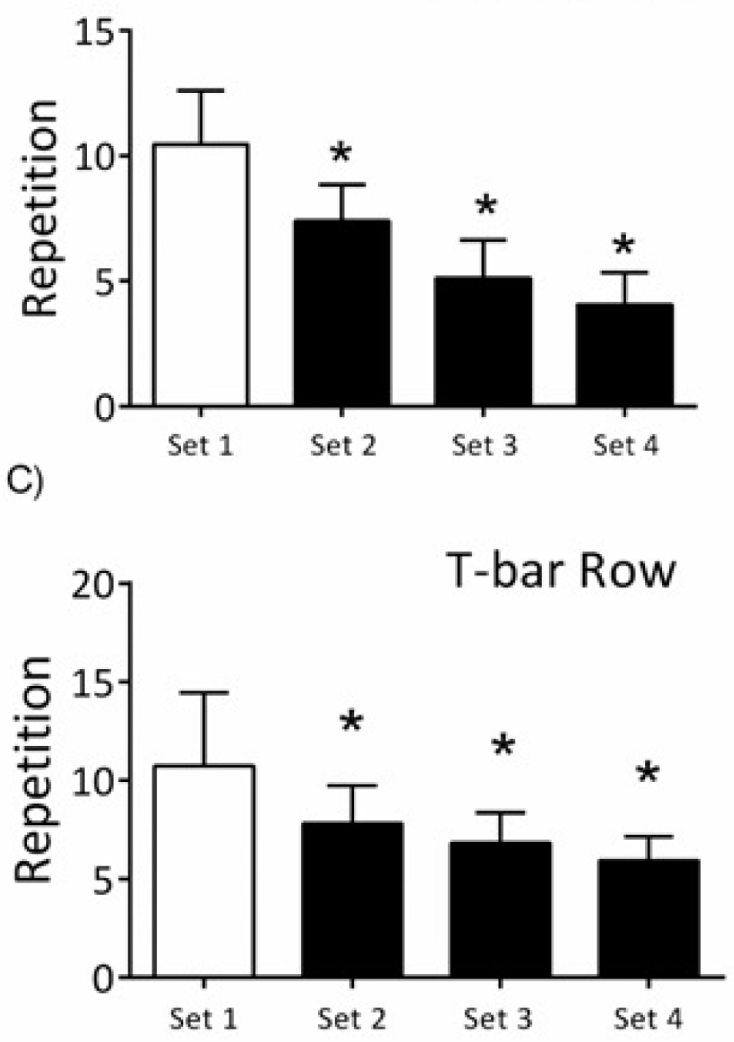

Figure 1 - Number of repetitions of the exercises performed in the acute session of strength training by the 15 participants. (70\% of 1RM). Data expressed as mean \pm SD values. Statistical analysis was performed using one-way ANOVA followed by the Tukey test. Value of * $\mathrm{p}<0.05$, between 1 st, 2 nd, $3 \mathrm{rd}$, and 4 th series. 
Table 1- A. Mean values of total intake (ml), Table 2-B, Ingested calories (Kcal) and Table 2-C, Time (minutes) at rest (day 0), or after exercise (day 14) during the drink test. Data are expressed as mean \pm SD, Percentile (25\% and $75 \%$ ), and 95\% CI (lower and upper). Statistical values were computed by the Student $\mathrm{t}$-test for paired data. ${ }^{*} \mathrm{p}<0.05 \mathrm{vs}$. rest.

(A) Total Intake (mL)

\begin{tabular}{|c|c|c|c|c|c|c|c|c|c|c|c|}
\hline \multirow[b]{3}{*}{ Mean } & \multicolumn{5}{|c|}{ Rest } & \multicolumn{6}{|c|}{ Exercise } \\
\hline & \multicolumn{3}{|c|}{ Percentile } & \multicolumn{2}{|c|}{$95 \%$ CI of mean } & \multirow[b]{2}{*}{ Mean } & \multirow[b]{2}{*}{ SD } & \multicolumn{2}{|c|}{ Percentile } & \multicolumn{2}{|c|}{$95 \%$ CI } \\
\hline & SD & $25 \%$ & $75 \%$ & Lower & Upper & & & $25 \%$ & $75 \%$ & Lower & Upper \\
\hline 1410 & 424 & 1024 & 1721 & 1141 & 1679 & $1866 *$ & 532 & 1489 & 2396 & 1529 & 2204 \\
\hline
\end{tabular}

(B) Ingested Calories (Kcal)

\begin{tabular}{|c|c|c|c|c|c|c|c|c|c|c|c|}
\hline \multicolumn{6}{|c|}{ Rest } & \multicolumn{6}{|c|}{ Exercise } \\
\hline \multirow[b]{2}{*}{ Mean } & \multirow[b]{2}{*}{ SD } & \multicolumn{2}{|c|}{ Percentile } & \multicolumn{2}{|c|}{$95 \%$ CI of mean } & \multirow[b]{2}{*}{ Mean } & \multirow[b]{2}{*}{ SD } & \multicolumn{2}{|c|}{ Percentile } & \multicolumn{2}{|c|}{$95 \%$ CI } \\
\hline & & $25 \%$ & $75 \%$ & Lower & Upper & & & $25 \%$ & $75 \%$ & Lower & Upper \\
\hline 985.9 & 345.4 & 688.7 & 1138 & 777.2 & 1195 & $1193 *$ & 384.7 & 928.1 & 1549 & 961 & 1426 \\
\hline
\end{tabular}

(C) Total Time (min)

\begin{tabular}{ccccccccccccc}
\hline \multicolumn{1}{c}{ Rest } & & \multicolumn{3}{c}{ Exercise } \\
\hline \multicolumn{1}{c}{} & \multicolumn{2}{c}{ Percentile } & \multicolumn{2}{c}{$\mathbf{9 5 \%}$ CI of mean } & & & \multicolumn{2}{c}{ Percentile } & 95\% CI \\
\hline Mean & SD & $\mathbf{2 5 \%}$ & $\mathbf{7 5 \%}$ & Lower & Upper & Mean & SD & $\mathbf{2 5 \%}$ & $\mathbf{7 5 \%}$ & Lower & Upper \\
$\mathbf{1 0 4 . 4}$ & 34.6 & 72 & 121 & 85.2 & 123.6 & $\mathbf{1 3 7 . 6 *}$ & 53.9 & 76 & 185 & 107.7 & 167.5 \\
\hline
\end{tabular}

Table 2 - Mean values of pro and anti-inflammatory markers at rest and after exercise. Data expressed as mean \pm SD. P-values or significance levels * ( $\mathrm{p}<0.05$ ) for IL-6, IL-10, IL-13, and TNF- $\alpha$ (I can't take it anymore) and IL-1 $\beta$ and IL-6 (I don't feel anything) vs. baseline, applying Student's t-test ( $\mathrm{n}=13$ ). Subtitle: IL-1 $\beta$, IL-6, IL-10, IL-13 and TNF- $\alpha$ (interleukins 1 beta, 6, 10, 13, tumor necrosis factor-alpha); ("I can't take it anymore" rest / "I can't take it anymore" exercise) pg / $\mathrm{mL}$ (picograms per milliliter).

\begin{tabular}{|c|c|c|c|c|}
\hline & \multicolumn{2}{|c|}{ Rest } & \multicolumn{2}{|c|}{ Exercise } \\
\hline & \multicolumn{4}{|c|}{ Pro-inflammatory cytokines } \\
\hline & (Score 0) & (Score 5) & (Score 0) & (Score 5) \\
\hline IL-1 $\beta(\rho g / m L)$ & $0.720 \pm 0.08$ & $0.864 \pm 0.09$ & $0.953 \pm 0.09 *$ & $0.683 \pm 0.12$ \\
\hline IL-6 ( $\rho g / m L)$ & $2.481 \pm 0.29$ & $3.858 \pm 0.40$ & $3.607 \pm 0.41 *$ & $2.528 \pm 0.40 *$ \\
\hline \multirow[t]{2}{*}{$\mathrm{TNF}-\alpha(\rho \mathrm{g} / \mathrm{mL})$} & $14.36 \pm 2.4$ & $17.94 \pm 2.8$ & $17.71 \pm 2.1$ & $9.521 \pm 1.4 *$ \\
\hline & \multicolumn{4}{|c|}{ Anti-inflammatory cytokines } \\
\hline IL-10 ( $\mathrm{g} / \mathrm{mL})$ & $1.362 \pm 0.14$ & $1.916 \pm 0.16$ & $1.542 \pm 0.19$ & $1.260 \pm 0.24 *$ \\
\hline IL-13 ( $\rho g / m L)$ & $0.9985 \pm 0.16$ & $1.069 \pm 0.12$ & $0.8977 \pm 0.09$ & $0.686 \pm 0.06 *$ \\
\hline
\end{tabular}


Table 3 - Linear regression analysis for CK, blood lactate, pro- and anti-inflammatory cytokines and total intake at score 0 and score 5 . * Significance was found for blood lactate at score 0 and CK at score 5 with $\mathrm{p}<0.05$ significance level. Subtitle: CK (creatinine kinase); Score 5 ("I can't take it anymore" rest / "I can’t take it anymore" exercise) pg / mL (picograms per milliliter); mmol/L (millimole per liter); U / L (unit per liter).

\begin{tabular}{|c|c|c|c|c|c|c|c|c|}
\hline \multirow{3}{*}{ Parameters } & \multicolumn{4}{|c|}{ Rest } & \multicolumn{4}{|c|}{ Exercise } \\
\hline & \multicolumn{2}{|c|}{ (Score 0) } & \multicolumn{2}{|c|}{ (Score 5) } & \multicolumn{2}{|c|}{ (Score 0) } & \multicolumn{2}{|c|}{ (Score 5) } \\
\hline & $\mathbf{R}^{2}$ & $\mathbf{p}$ & $\mathbf{R}^{2}$ & $\mathbf{p}$ & $\mathbf{R}^{2}$ & $\mathbf{p}$ & $\mathbf{R}^{2}$ & $\mathbf{p}$ \\
\hline CK (U/L) & 0.127 & 0.312 & 0.047 & 0.519 & 0.125 & 0.316 & 0.519 & $0.012 *$ \\
\hline Lactate $(\mathrm{mmol} / \mathrm{L})$ & 0.038 & 0.612 & 0.337 & 0.101 & 0.758 & $0.011 *$ & 0.038 & 0.642 \\
\hline \multicolumn{9}{|c|}{ Pro-inflammatory cytokines } \\
\hline IL-1ß $(\rho g / m L)$ & 0.011 & 0.771 & 0.025 & 0.658 & 0.004 & 0.860 & $<0.001$ & 0.942 \\
\hline IL-6 $(\rho g / m L)$ & 0.069 & 0.461 & 0.018 & 0.711 & 0.071 & 0.456 & 0.070 & 0.458 \\
\hline TNF- $\alpha(\rho g / m L)$ & 0.038 & 0.590 & 0.061 & 0.490 & 0.027 & 0.646 & 0.031 & 0.627 \\
\hline \multicolumn{9}{|c|}{ Anti-inflammatory cytokines } \\
\hline IL-10 ( $\mathrm{gg} / \mathrm{mL})$ & 0.036 & 0.591 & 0.003 & 0.884 & 0.005 & 0.840 & 0.012 & 0.759 \\
\hline IL-13 ( $\mathrm{gg} / \mathrm{mL})$ & 0.002 & 0.910 & 0.069 & 0.462 & 0.991 & $<0.001$ & 0.002 & 0.893 \\
\hline
\end{tabular}

\section{Discussion}

In the present study, acute-SE promoted an increase in GA as well as altered biochemical parameters and plasma cytokine levels. In relation to the anthropometric parameters, the studied group had an average BMI classified as excessive weight, but the body fat percentage was within the reference values proposed in the literature ${ }^{21}$. The characterization as the excessive weight was probably due to the high values of muscle mass in this population because they all engaged in strength training. The basal metabolic rate was similar to that obtained in the study of Sharp et al. ${ }^{22}$, who also found mean values of about $1700 \mathrm{kcal} /$ day in white and African American young men. It is possible to affirm that these results are directly influenced by their fat-free mass.

The results of the 1-RM test showed low mean values for the squat exercise compared with the data obtained by Pearcey et al. ${ }^{23}$, who studied a similar population of young men. For the bench press exercise, the results were similar to those obtained by Calatayud et al. ${ }^{24}$ and higher than the values observed for bench press and T-bar row exercises performed by young men studied ${ }^{20}$.

During the series of acute-SE until muscular exhaustion, a decrease in the number of repetitions throughout the series was observed. Muscle fatigue can occur due to changes in homeostasis in the skeletal muscle itself or alterations in the neural input that reaches the muscle, resulting in a progressive decrease in the speed and frequency of repetition ${ }^{25}$. In addition, the sample evaluated in this study could perform more repetitions of the squat exercise than the other exercises.

CK concentrations have been used as markers of exercise-related stress ${ }^{26}$ and are causally related to muscle damage as well as lactate levels ${ }^{27}$. CK concentration is individually and markedly elevated 1-4 days after exercise. Thus, it is an indicator of an athlete's training and recovery status ${ }^{25}$. In the present study, we observed that the increase $\mathrm{CK}$ response accelerated during the acute-SE session compared to the rest state in the first score. The rise of CK levels can be a positive adaptive response to induced muscle damage, and its levels remain high in subjects adapted to $\mathrm{SE}^{26}$.

Similarly, we observed a significant increase in blood lactate during exercise compared to the rest state, which seems to be an effect of the acute-SE training, as previously reported in the literature ${ }^{28}$. However, the ingestion of the chocolate milk beverage did not affect blood CK and lactate levels.

A significant decrease in glucose concentrations was also observed after exercise at the end of the drink test, which can be a response to an acute-SE stimulus. A possible explanation for this result is the rise in glucose uptake due to increased activity of glucose transporter proteins (GLUTs) in response to increased AMP concentrations, commonly observed as an adaptation to training ${ }^{29}$. Kyröläinen et al. ${ }^{30}$ also observed that SE diminished glucose concentrations in healthy young women as we observed in our study.

Concerning the influence of exercise on GA, an increase in total intake, ingested calories, and total time in males was observed after the acute-SE session. These data demonstrated that acute-SE promotes increases in GA and decreases satiety in healthy males. A possible explanation for these results is that acute-SE leads to the activation of the vagal cholinergic pathway, which in turn increases gastric tone and the release of nitric oxide, promoting $\mathrm{GA}^{31}$. In addition, the varied and contradictory responses to the effects of different intensities of physical exercise on food intake and energy intake can be explained by 
differences in sex and nutritional and fitness statuses ${ }^{32}$.

Our data showed that acute-SE also appears to increase the serum levels of inflammatory markers such as IL-6, which can influence GA and appetite. IL-6 is secreted during exercise in a contraction-dependent manner and can be related to the release of appetite hormones, such as ghrelin, GLP-1, and PYY, which influence food intake. However, the role of cytokines in the secretion of these hormones is not well understood ${ }^{15}$.

In our study, acute-SE modified the plasma cytokine profile. These results are similar to those observed by Ihalainen et al..$^{33}$, who reported an increase in the circulating concentrations of IL-1 $\beta$, IL-6, IL-1ra, and resistin. The increase in cytokine concentrations is an adaptive response to the elevated production of reactive oxygen species generated by the respiratory chain during exercise as well as the immune response, causing muscle damage and microlesions generated by acute-SE, which are directly affected by the physiological demands, volume, load, and intensity of the exercise ${ }^{34}$. At the score of 0 in the drink test, an increase in IL-1 $\beta$ was noticed after the acute-SE session. These findings corroborate the results obtained by Rosa Neto et al. ${ }^{35}$, who reported that a single moderate-to-high intensity resistance exercise can promote an increase in the levels of IL-6 and IL- $1 \beta$ cytokines.

An increase in IL-6 levels was observed immediately after the training at the 0 score and a significant reduction occurred at the last score. A possible explanation for these effects is that muscles secrete IL- 6 to allow the body to maintain its energy status during exercise by acting as an energy sensor to contract muscles and stimulate glucose production. In the recovery phase, with adequate rest and nutrition (glucose ingestion during the drink test), the IL-6 response to exercise can be attenuated ${ }^{36}$. Forti et al. ${ }^{37}$ also observed a decrease in IL-6 concentrations after SE. However, more research is needed to comprehend the behavior of IL-6 in exercise and its influence on energy status.

During exercise, the secretion of IL- 6 also stimulates the production of anti-inflammatory cytokines, such as IL-10. Therefore, if there is an increase in IL-6 concentrations, IL-10 also increases, and if there is a decrease in IL- 6 concentrations, IL-10 secretion also decreases ${ }^{38}$. These data were confirmed in our study by the correlation analysis, which showed a positive result between IL- 6 and IL-10 at two points of the drink test, but only after acute-SE.

The anti-inflammatory cytokines IL-10 and IL-13 seem to be upregulated after acute-SE ${ }^{36}$, but we observed a decrease in IL-10 and IL-13 concentrations after acute-SE. The decrease in anti-inflammatory IL-13 can also be related to the attenuated inflammatory response observed at score 5 seen in this study, as occurred with IL-10 cytokine, mentioned previously.

In relation to the response of $\mathrm{TNF}-\alpha$, its levels were lower at score 5 compared with that at the basal time score, thus displaying a profile similar to other pro-inflammatory cytokines of this study, such as IL-6. These results can be related to an initial increase of the IL-6 cytokine level, which may act to suppress the release of TNF- $\alpha$ by stimulating the expression of anti-inflammatory cytokines like IL-10 and IL-1 $\mathrm{ra}^{39}$.

We also investigated the interplay of CK, lactate, cytokine profile, and total intake or GA at each score of the drink test.
A correlation was found for $\mathrm{CK}$ at score 5 , for lactate at score 0 , and cytokines IL- $6,-13$, and TNF- $\alpha$ and volume ingested at score 5. These results reinforce the role of exercise in the control of GA. Both CK and lactate are markers related to the intensity of exercise, in turn, related to mechanisms of gastric emptying and accommodation ${ }^{8}$. In the sense, Islam et al. ${ }^{15}$ found that blood lactate was correlated to the area under the curve (AUC) values for ghrelin and IL-6 to AUC values for GLP-1, both with hormones involved in appetite control. Regarding TNF- $\alpha$, its relationship can be explained by its role in the secretion of the other pro-inflammatory cytokines ${ }^{39}$.

\section{Conclusion}

In the study, we showed that acute strength exercise induces a decrease in gastric satiety. In addition, acute strength exercise alters some biochemical parameters as well as the plasma cytokine levels. We suggest more studies to clarify other possible mechanisms involving acute strength exercises and gastric function

\section{References}

1. Verburgh L, Königs M, Scherder EJ, Oosterlaan J. Physical exercise and executive functions in preadolescent children, adolescents, and young adults: a meta-analysis. Br J Sports Med. 2014; 48(12):973-9.

2. McLaughlin M, Jacobs I. Exercise Is Medicine, But Does It Interfere with Medicine? Exerc Sport Sci Ver. 2017;45(3): 127-135.

3. Krogh K, Chiarioni G, Whitehead W. Management of chronic constipation in adults. United Eur Gastroent. 2017; 5(4):465-472.

4. Silva MT, Palheta-Junior RC, Sousa DF, Fonseca-Magalhães PA, Okoba W, Campos CPS et al. Sodium bicarbonate treatment prevents gastric emptying delay caused by acute exercise in awake rats. J Appl Physiol (1985). 2014; 1;116(9):1133-41.

5. Meyer-Gerspach AC, Wölnerhanssen B, Beglinger B, Nessenius F, Napitupulu M, Shulte FH, et al. Gastric and intestinal satiation in obese and normal weight healthy people. Physiol Behav. 2014; (22)129: 265-71.

6. Hazell TJ, Islam H, Townsend LK, Schmale MS, Copeland JL. Effects of exercise intensity on plasma concentrations of appetite-regulating hormones: potential mechanisms. Appetite. 2016; 1(98): 80-8.

7. Müller IA, Wedell-Neergaard AS, Solomon TPJ, Karstoft K. The impact of acute bouts of interval and continuous walking on energy $\square$ intake and appetite regulation in subjects with type 2 diabetes. Physiol Rep. 2017;5(23):e13524.

8. Horner KM, Schubert MM, Desbrow B, Byrne NM, King NA. Acute exercise and gastric emptying: a meta-analysis and implications for appetite control. Sports Med. 2015; 45(5):659-78.

9. Beattie K, Kenny IC, Lyons M, Carson BP. The effect of strength training on performance in endurance athletes. Sports Med. 2014; 44(6):845-65. 
10. Brandt $\mathrm{C}$, Pedersen BK. The role of exercise-induced myokines in muscle homeostasis and the defense against chronic diseases. J Biomed Biotechnol. 2010; 5: 20258.

11. Baird MF, Graham SM, Baker JS, Bickerstaff GF. Creatinekinase- and exercise-related muscle damage implications for muscle performance and recovery. Nutr Metab. 2012; 2012:960363.

12. Cerqueira E, Marinho DA, Neiva HP., Lourenço, O. Inflammatory Effects of High and Moderate Intensity Exercise-A Systematic Review. Front Physiol 2020 9;(10):1550.

13. Shi Y, Shi H, Nieman DC, Hu Q, Yang L, Liu T, et al. Lactic Acid Accumulation During Exhaustive Exercise Impairs Release of Neutrophil Extracellular Traps in Mice. Front Physiol. 2019; 12;10:709.

14. Engevik AC, Kaji I, Goldenring JR. The Physiology of the Gastric Parietal Cell. Physiol Rev. 2020;100(2):573-602.

15. Islam H, Townsend LK, McKie GL, Medeiros PJ, Gurd BJ, Hazell TJ. Potential involvement of lactate and interleukin-6 in the appetite-regulatory hormonal response to an acute exercise bout. J Appl Physiol (1985). 2017;123(3):614-623.

16. Pedersen, B. K. Anti-inflammatory effects of exercise: role in diabetes and cardiovascular disease. Eur J Clin Invest. 2017; 47(8):600-611.

17. Peake JM, Neubauer O, Della Gatta PA, Nosaka K. Muscle damage and inflammation during recovery from exercise. J Appl Physiol. (1985). 2017;122(3):559-570.

18. Lang LL, Lyngbaek MP, Soederlund L, Legaard GE, Ehses JA, Haywwod SE, et al. Interleukin-6 Delays Gastric Emptying in Humans with Direct Effects on Glycemic Control. Cell Metab. 2018; 5;27(6):1201-1211.

19. Inada T, Hamano N, Yamada M, Shirane A, Shingu K. Inducible nitric oxide synthase and tumor necrosis factor-alpha in delayed gastric emptying and gastrointestinal transit induced by lipopolysaccharide in mice. Braz J Med Biol Res. 2006; 39(11):1425-34.

20. Agostinete RR, Rossi FE, Magalhaes AJ, Rocha AP, Parmezzani SS, Gerosa-Neto J, et al. Immunometabolic responses after short and moderate rest intervals to strength exercise with and without similar total volume. Front Physiol. 2016; $25 ;(7): 444$.

21. Bray GA. Contemporary diagnosis and management of obesity. Handbooks in Health Care Company 1998.

22. Sharp TA, Bell ML, Grunwald GK, Schmitz KH, Sidney $\mathrm{S}$, Lewis $\mathrm{CE}$, et al. Differences in resting metabolic rate between white and African-American young adults. Obes Res. 2002; 10(8):726-32.

23. Pearcey GE, Bradbury-Squires DJ, Kawamoto JE, Drinkwater EJ, Behm DG, Button DC. Foam rolling for delayed-onset muscle soreness and recovery of dynamic performance measures. J Athl Train. 2015; 50(1):5-13 50:5-13.

24. Calatayud J, Borreani S, Colado JC, Martin F, Tella V, Andersen LL. Bench press and push-up at comparable levels of muscle activity results in similar strength gains. J Strength Cond Res. 2015;29(1):246-53.

25. Párraga-Montilla JA, García-Ramos A, CastañoZambudio A, Capelo-Ramírez F, González-Hernández JM,
Cordeiro-Rodriguez Y, et al. Acute and delayed effects of a resistance training session leading to muscular failure on mechanical, metabolic, and perceptual responses. J Strength Cond Res. 2020; 34(8):2220-2226.

26. Kay AD, Rubley B, Talbot C, Mina M, Baross AW, Blazevich AJ. Stretch imposed on active muscle elicits positive adaptations in strain risk factors and exercise-induced muscle damage. Scand J Med Sci Sports. 2018; 28(11):2299-2309.

27. Tajra V, Tibana RA, Vieira DC, de Farias DL, Teixeira TG, Funghetto SS, et al. Identification of high responders for interleukin- 6 and creatine kinase following acute eccentric resistance exercise in elderly obese women. J Sci Med Sport 2014; 17(6):662-6.

28. Cairns SP. Lactic acid and exercise performance: culprit or friend? Sports Med. 2006; 36(4):279-91

29. Sylow L, Kleinert M, Richter EA, Jensen TE. Exercisestimulated glucose uptake - regulation and implications for glycaemic control. Nat Rev Endocrinol. 2017; 13(3):133-148.

30. Kyröläinen H, Hackney AC, Salminen R, Repola J, Häkkinen $\mathrm{K}$, Haimi J. Effects of combined strength and endurance training on physical performance and biomarkers of healthy young women. J Strength Cond Res. 2018 32(6):1554-1561.

31. Ikeo K, Oshima T, Sei H, Kondo T, Fukui H, Watari J, et al. Acotiamide improves stress $\square$ induced impaired gastric accommodation. Neurogastroenterol Motil. 2017;29(4):e12991.

32. Bilski J, Mańko G, Brzozowski T, Pokorski J, Nitecki J, Nitecka A, et al. Effects of exercise of different intensity on gut peptides, energy intake, and appetite in young males. Ann Agric Environ Med 2013; 20 (4):787-93.

33. Ihalainen JK, Ahtiainen JP, Walker S, Paulsen G, Selänne $\mathrm{H}$, Hamalainen $\mathrm{M}$, et al. Resistance training status modifies the inflammatory response to explosive and hypertrophic resistance exercise bouts. J Physiol Biochem. 2017 73(4):595-604.

34. Kanda K, Sugama K, Hayashida H, Sakuma J, Kawakami $\mathrm{Y}$, Miura S, et al. Eccentric exercise-induced delayed-onset muscle soreness and changes in markers of muscle damage and inflammation. Exerc Immunol Rev 2013;19:72-85.

35. Rosa Neto JC, Lira FS, Zanchi NE, Oyama LM, Pimentel GD, Santos RVT, et al. Acute exhaustive exercise regulates IL-2, IL-4 and MyoD in skeletal muscle but not adipose tissue in rats. Lipids Health Dis. 2011; 13;10:97.

36. Hennigar SR, McClung JP, Pasiakos SM. Nutritional interventions and the IL-6 response to exercise. FASEB J. 2017: 31(9):3719-3728.

37. Forti LN, Van Roie E, Njemini R, Coudyzer W, Beyer I, Delecluse C, et al. Effects of resistance training at different loads on inflammatory markers in young adults. Eur J Appl Physiol 2017: 117(3):511-519.

38. Timmerman KL, Amonette WE, Markofski MM, Ansinelli HA, Gleason EA, Rasmussen BB, et al. Blunted IL-6 and IL10 response to maximal aerobic exercise in patients with traumatic brain injury. Eur J Appl Physiol 2015; 115(1):111-8.

39. Bilski J, Mazur-Bialy A, Brzozowski B, Magierowski M, Zahradnik-Bilska J, Wòjsik D, et al. Can exercise affect the course of inflammatory bowel disease? Experimental and clinical evidence. Pharmacol Rep 2016; 68(4):827-36. 


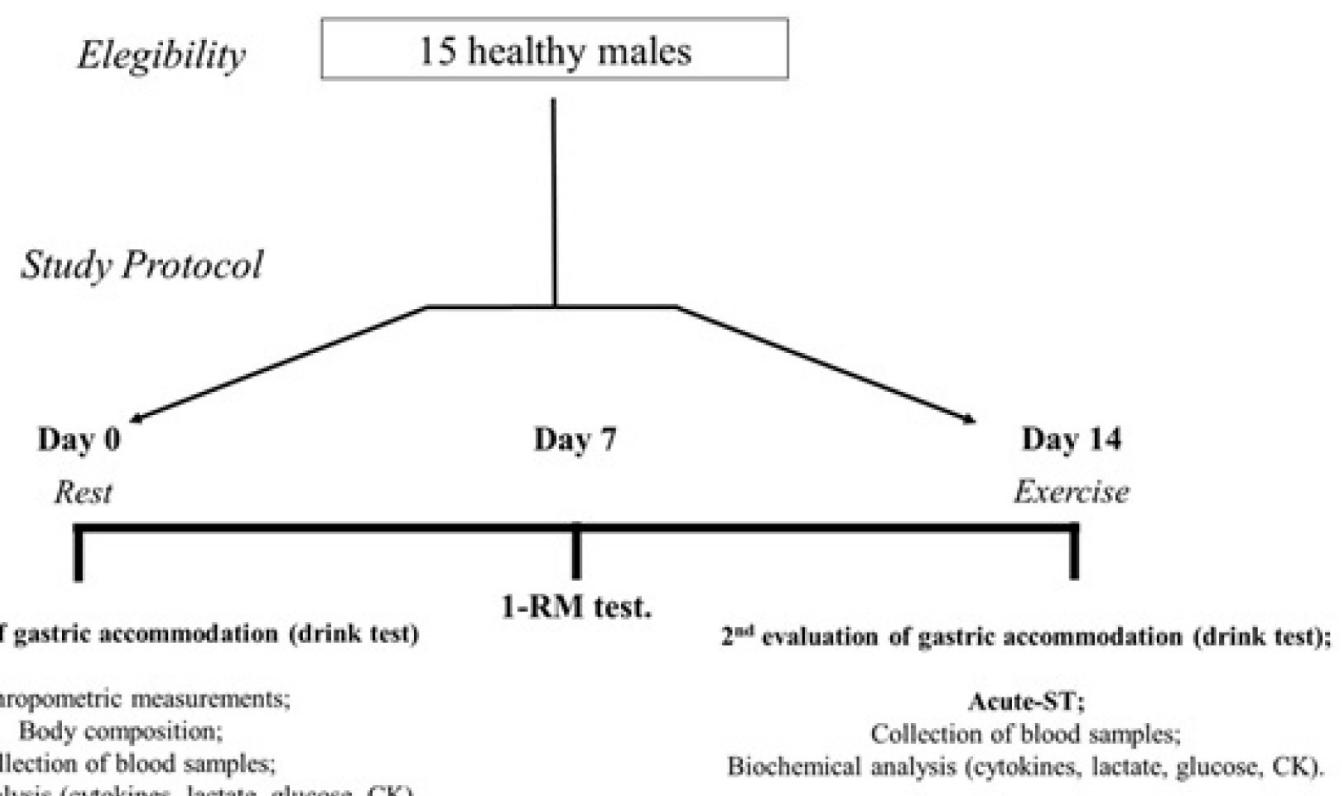

Biochemical analysis (cytokines, lactate, glucose, CK).

\section{Drink Test}

\section{Blood samples collect}
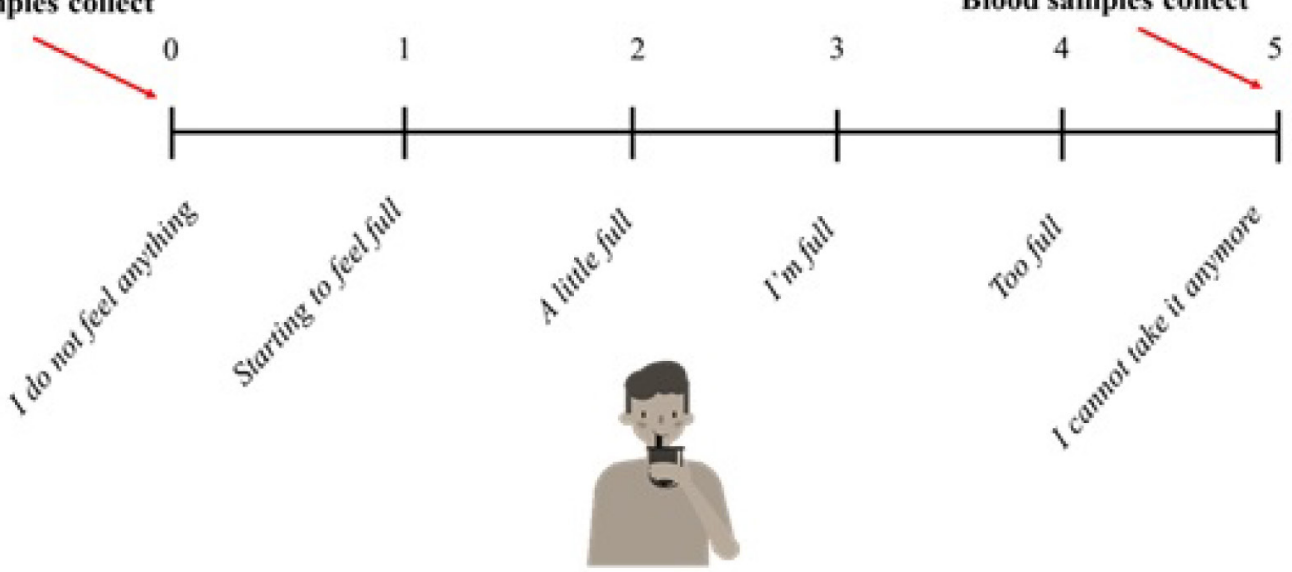

Supplementary materials S1

Summary of the study protocol.

Legend: 1-RM test: one maximum repetition test.; acute-SE: acute strength training.

\section{Acknowledgments}

It was supported by grants from the Coordination for the Improvement of Higher Education Personnel (Coordenação de Aperfeiçoamento de Pessoal de Nível Superior - CAPES), the National Council for Scientific and Technological Development (Conselho Nacional de Desenvolvimento Científico e Tecnológico - CNPq) and the Foundation for Scientific and Technological Development of the State of Ceará (Fundação Cearense de Apoio ao Desenvolvimento Científico e Tecnológico - Funcap).

\section{Corresponding author}

Prof. Dr. Moisés Tolentino

Federal University of Piaui. Health Sciences Center - Department of Physical Education. Laboratory of Exercise and Gastrointestinal Tract. Campus Ministro Petônio
Portela, sn, Bairro Ininga, Teresina, PI, Brazil CEP. 64049-550. Telephone: +5586 3237-2332.

Email: tolentino@ufpi.edu.br

Manuscript received on September 28, 2020.

Manuscript accepted on October 7, 2020.

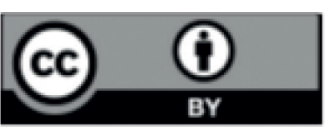

Motriz. The Journal of Physical Education. UNESP. Rio Claro, SP, Brazil - eISSN: 1980-6574 - under a license Creative Commons - Version 4.0 